\title{
On the Impact of the Primary Network Activity on the Achievable Capacity of Spectrum Sharing over Fading Channels
}

\author{
Mohammad G. Khoshkholgh, Keivan Navaie, Member, IEEE, and Halim Yanikomeroglu, Member, IEEE
}

\begin{abstract}
When utilizing spectrum sharing in wireless channels, a secondary service may access the spectrum allocated to the primary service while this frequency band is under-utilized. The availability of the frequency band to the secondary user is a function of the activity of the users in the primary network. In this paper, we analyze the achievable capacity of the secondary service which employs opportunistic spectrum Access (OSA) over a fading environment based on the primary network activity. We categorized OSA methods into Access Limited OSA (ALOSA), and Interference Limited OSA (IL-OSA) schemes. In AL-OSA the spectrum is shared with the secondary service in circumstances in which the primary service is totally inactive however, in IL-OSA access to the spectrum is allowed subject to an interference threshold. For both cases we develop analytical frameworks to analyze the impact of the primary network activity on the achievable capacity of the secondary service. Simulation results confirm our analysis and also show that in cases where higher activity is in the primary network, IL-OSA is the more appropriate OSA method. For a less active primary network, AL-OSA is shown to performs better with respect to the achievable capacity.
\end{abstract}

Index Terms-DS-CDMA networks, OFDM, opportunistic spectrum access, spectrum sharing.

\section{INTRODUCTION}

$\mathbf{S}$ PECTRUM SHARING was first proposed by the Federal Communications Commission (FCC) [2] to improve the utilization of the allocated frequency bands. In this method, under certain conditions, a Secondary Service is able to access to a frequency band formally allocated to the Primary Service [3]. Various schemes are proposed in the literature for spectrum sharing (see e.g., [4] and references therein). In this paper, our focus is the Opportunistic Spectrum Access (OSA).

In OSA the secondary service dynamically detects and makes use of the spectrum holes [5]. Spectrum holes are those parts of the spectrum allocated to the primary user which are under-utilized in some particular times and specific locations.

Manuscript received April 23, 2008; revised September 2, 2008; accepted December 26, 2008. The associate editor coordinating the review of this paper and approving it for publication was M. Guizani.

M. G. Khoshkholgh and K. Navaie are with the Department of Electrical and Computer Engineering, Tarbiat Modares University, Tehran, Iran, P. O. Box 14155-4843 (e-mail: keivan@sce.carleton.ca).

$\mathrm{H}$. Yanikomeroglu is with the Broadband Communications and Wireless Systems (BCWS) Centre, Department of Systems and Computer Engineering, Carleton University, Ottawa, ON, Canada, K1S 5B6 (e-mail: halim@sce.carleton.ca).

Part of this paper has been presented at the IEEE 68 Vehicular Technology Conference: VTC2008-Fall, September 2008, Calgary, Canada [1].

Digital Object Identifier 10.1109/TWC.2009.080562
Clearly, spectrum sharing must not cause Quality-of-Service degradation to the primary service.

In the spectrum sharing, the under-utilized spectrum as the available radio resource which can be utilized by the secondary service varies over time. Given an acceptable level of accuracy in the spectrum sensing, the temporal variations can then be exploited subject to the primary system interference threshold. Therefore, the maximum achievable capacity is directly affected by the temporal variations in the available radio resources. It is shown in [6] and [7] that obtaining the maximum achievable capacity for an Additive White Gaussian Noise (AWGN) channel in different transmission scenarios with interference threshold is an instant of the water filling problem.

Under various fading conditions, the achievable capacity is studied in [8]. It is shown in [8] that employing OSA results in a significant increase in the achievable capacity in comparison with the OSA in an equivalent AWGN channel. However, in [8] a constant level of activity is assumed for the primary network thus the available radio resource is assumed to be constant.

Different parameters have impacts on the temporal variation of the available radio resource in a spectrum sharing system including the dynamic behavior of wireless channels as well as the activity of the users in the primary network. User activity in the primary network includes two important parameters: the number of active users, and their corresponding traffic profile.

In most of the previous works, the primary users' activity is simply ignored by assuming constant bit-rate transmission. However, in practise most of the data services are bursty in nature; therefore, the constant activity assumption is questionable. In other words, the activity of the users in the primary networks have impact on the fraction of time (or the level of transmission power) in which the secondary service is able to access the spectrum, thus on the achievable capacity. In this paper, we investigate the impact of the primary users' activity on the secondary service achievable capacity.

In our model of the OSA system, the spectrum is divided into a number of sub-channels and then the secondary service makes access to one (or more) of these sub-channels, subject to the interference threshold constraint. Generally, two types of OSA can be envisaged. First, the OSA in which by adopting an appropriate method (see e.g., [4]) the secondary service detects the time-intervals in which the primary service is totally inactive. In such cases, the main constraint which is imposed 
on the achievable capacity is the available access time. We refer to this scenario as Access-Time Limited OSA (AL-OSA). The second method is the one in which the secondary service is able to access the spectrum currently in use while the primary service is also active subject to complying with the interference threshold. In this case however, the temporal interference level in the receiver of the primary service is the main player. We refer to this scenario as Interference Limited OSA (IL-OSA).

In AL-OSA the secondary service should be able to make an accurate estimation of the time intervals in which the frequency band (or a part of it) is free, i.e., not used by the primary service [7]. The more communication activity in the primary network, the less the probability of spectrum availability to the secondary service is. Similar to the previous works (see, e.g., [9], [10], [11]), to model the spectrum availability, we utilize an ON/OFF model. We then analyze the achievable capacity for AL-OSA in Section III after describing the system model in Section II. Our analytical framework captures the dependency between the achievable capacity and the activity of the primary network. We also obtain a closed-form for the achievable capacity which indicates its dependency to the primary network activity. The impact of inaccurate estimation of sub-channel availability is also investigated. AL-OSA is an example of OSA system where both primary and secondary services make use of Orthogonal Frequency Division Multiplexing (OFDM).

In IL-OSA the secondary service is able to make access to the frequency band (or a part of it) while the channel is used by the primary service, whereby an appropriate mechanism is adopted to ensure that the primary service access to the spectrum is not degraded. To quantify the impact of the spectrum sharing on the primary network, the FCC introduced Interference Temperature as a metric: the secondary service is able to access a certain frequency band if the interference temperature constraint is satisfied [12], [13]. This metric, however is not further considered as a base for the standardization activities by the FCC [14]. In this paper, as a metric for recognizing a spectrum hole we consider a threshold on the acceptable level of the imposed interference caused by the operation of the secondary user. Therefore, a spectrum hole is defined as a frequency band in which the interference level is below the Interference Threshold.

We also propose an analytical framework and obtain the achievable capacity for IL-OSA in Section IV based on the primary network activity. We then obtain a closed-form for the achievable capacity that indicates its dependency on the primary network activity. A very important aspect of our analysis is that we modelled the impact of the primary network interference on the achievable capacity of the secondary service. IL-OSA is an appropriate scheme for cases where the primary service is utilized an interference limited air interface technology such as Direct Sequence Code Division Multiple Access (DS-CDMA).

DS-CDMA is the dominant air interface technique for the third generation $(3 \mathrm{G})$ mobile communications and some Wireless Local Area Network (WLAN) technologies. Therefore, the spectrum sharing over existing DS-CDMA-based networks is anticipated to be one of important spectrum sharing applica- tions in the near future. In DS-CDMA the system performance is limited by the maximum total received interference. The total interference, among other things, is a function of the primary users' communication activity. In OSA, part of the maximum acceptable interference is considered to be created by the secondary service. Here, we call this portion the interference threshold. Therefore, decreasing the activity of the primary users results in a higher interference threshold while creating less interference at the secondary service receiver. Thus the achievable capacity of the secondary service is increased. Generally, utilizing OFDM by the secondary service provides the required flexibility for the secondary service in terms of the access over appropriate portions of the spectrum [12].

A direct consequence of the analytical results presented in this paper is that adopting a method by the secondary service to efficiently exploit the temporal variations of the available radio resource (i.e., accessible sub-channel in AL-OSA and acceptable interference in IL-OSA ), can significantly increase the achievable capacity of OSA systems. The temporal variations are mainly due to the traffic characteristics of the primary users as well as their corresponding wireless channel temporal dynamics, which are both considered in our formulations.

We then simulate the performance of AL-OSA and IL-OSA systems. In our simulations we use a practical channel model which is presented in Section V. The simulation results are then presented in Section VI which confirm the analytical derivations obtained from our proposed framework. This paper is concluded in Section VII, where we also present some practical considerations.

The main contributions of this paper are as follows. We propose a mathematical framework to model the impact of the primary network activity on the maximum achievable capacity in OSA for IL-OSA and Al-OSA scenarios. We also conclude that under the equivalent network conditions for high (low) primary network activity, the achievable capacity for the ILOSA is higher (lower) than that of AL-OSA. The results obtained in this paper shed light on the dependency between the achievable capacity and the primary network activity.

\section{System Model}

In spectrum sharing, two services try to access a $B \mathrm{~Hz}$ spectrum band: the Primary Service and the Secondary Service. The frequency band has been licensed to the primary service. The primary service is provided by the Primary Network to the Primary Users. The secondary service does not have the spectrum license, but may acquire access to the spectrum by adopting OSA. The secondary service utilizes OFDM to access the spectrum. OFDM provides the required flexibility to the secondary service to access separate under-utilized portions of the spectrum band [12].

In this paper we focus on the impact of the primary service activity on the maximum achievable capacity of the secondary service. Therefore, for brevity of expositions, we consider one secondary service user, which is simply referred to as secondary user. This assumption is taken in the literature (see e.g., [6], [7] and [8]). Of course in cases where number of secondary users is more than one, depending on the access 
strategy, the inter-operation among the secondary users may reduce the achieved capacity, thus the obtained capacity bound remains valid.

We consider a frequency-selective wireless channel with AWGN. The coherence bandwidth of the channel is $B_{c} \mathrm{~Hz}$. The white noise power spectral density is $N_{0}$. The channel is divided into $N$ flat fading $B_{c} \mathrm{~Hz}$ sub-channels. Sub-channels are indexed by $i=1,2, \ldots, N$. Based on the primary network status, the secondary service may have access to $M$ accessible sub-channels indexed by $j=0,1, \ldots, M$ where $0 \leq M \leq N$. Based on the adopted OSA scenario, the number of accessible sub-channels to the secondary service is either estimated adaptively based on the network status, or pre-assigned by the primary network or the regulator.

The sub-channel gains are independent and identically distributed (i.i.d.) random processes. Subscripts $s$ and $p$ are used in this paper for the secondary service and the primary service, respectively. Hereafter, we simply refer to "primary spectrum" as "spectrum" unless otherwise stated.

The operation of the secondary service in the spectrum implies interference on the primary service users. The interference threshold, $Q$, is the maximum allowable average interference in the receiver of the primary service caused by the operation of the secondary service in the spectrum.

In AL-OSA, the spectrum sharing exploits idle sub-channels for the secondary service transmission. In this case, given an accurate spectrum sensing procedure, AL-OSA utilizes the un-used capacity of the primary network, without impeding the concurrent active primary service users. However, for an inaccurate spectrum sensing technique, AL-OSA may cause interference on the active sub-channels of the primary network, and subsequently reduces the QoS of those sub-channels. In this case, the imperfectness of the spectrum sensing may reduce the actual capacity of the primary network where the amount of this reduction is acceptable for the primary service. In practice, the effect of the inaccurate spectrum sensing on the capacity is modelled by the probability of collision between the primary service and the secondary service data (see Subsection III-B).

In IL-OSA, the spectrum sharing is based on $Q$, which is determined by either regulator, or the primary network operator so that the QoS constraints of the primary services are guaranteed. Therefore, the un-used capacity of the primary network is exploited by spectrum sharing without degrading the QoS, or decreasing the primary network capacity.

Fig. 1 shows the schematic diagram of the system considered in this paper. For sub-channel $i, g_{0 i}$ and $g_{1 i}$ in Fig. 1 denote the instantaneous power gains of sub-channel $i$ from the secondary transmitter to the primary and the secondary receivers, respectively. Both $g_{0 i}$ and $g_{1 i}$ are assumed to be stationary and ergodic independent random variables with unit-mean probability density functions (pdf) ${ }^{1}, f_{0 i}\left(g_{0 i}\right)$, and, $f_{1 i}\left(g_{1 i}\right)$, respectively which are i.i.d. for $i=1,2, \ldots, N$. We also assume that $F_{0 i}(x), F_{1 i}(x)$ are probability distribution function (PDF) of $g_{0 i}$ and $g_{1 i}$, respectively.

Since the main objective of this paper is to analyze the

\footnotetext{
${ }^{1}$ The unit mean assumption is for brevity of expositions. We consider a general channel model later in Section V.
}

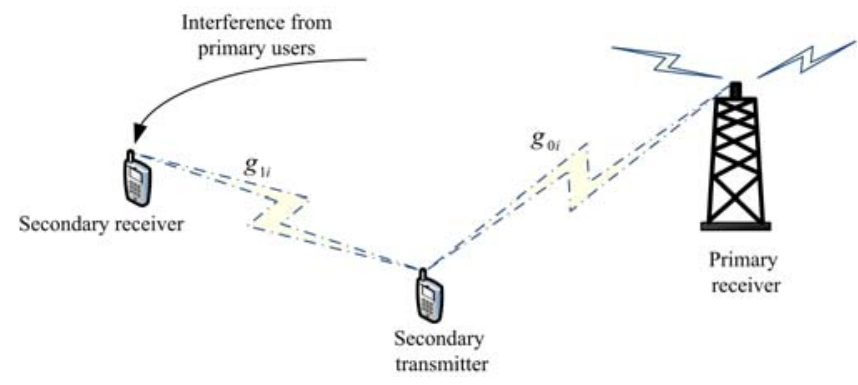

Fig. 1. Spectrum sharing structure for sub-channel $i$.

impact of the primary users' activity on the maximum achievable capacity, we assume the ideal case where a spectrum coordinator exists and coordinates spectrum access among the primary and the secondary networks. The spectrum coordinator enables the primary and the secondary transmitters to have perfect Channel Side Information (CSI) pair $\left(g_{0 i}, g_{1 i}\right)$, for $i=1, \ldots, N$.

Generally, the instantaneous transmit power of the secondary transmitter in the $i$ th sub-channel is $P_{s i}\left(g_{0 i}, g_{1 i}\right)$ which is a function of both $g_{0 i}$ and $g_{1 i}$. The maximum transmit power of the secondary transmitter is $\bar{P}_{s}$. To obtain the maximum achievable capacity we also assume that upon accessibility of the spectrum there is always enough backlogged traffic in the secondary service which is ready for transmission.

\section{A. Users' Activity in the Primary Network}

As it was mentioned in Section I the available radio resource for spectrum sharing is a function of users' traffic in the primary network characteristics. The term traffic characteristics in a wireless network is usually referred to the statistics of the number of active users as well as their communication activity during a call or a session. Given this, in this paper the traffic characteristics of the primary users is modelled by user's activity. Thus, the term "activity" refers to the fraction of time in which an individual primary user transmits.

1) AL-OSA System: To study the impact of traffic characteristics on the AL-OSA system, we consider an OFDM primary network with independent flat fading $B_{c} \mathrm{~Hz}$ sub-channels. We assume that the primary network adaptively allocates the subchannels to the users. Therefore when there is no traffic to be transmitted, some sub-channels are available to be utilized by the secondary service. Since the maximum achievable capacity is the subject of the study, in this paper, here we assume that sub-channel availability is accurately estimated by the secondary service.

Availability of one or more sub-channels for spectrum sharing, among other things, is a function of the traffic characteristic of the primary users; the more the activity of the users in the primary network, the less available number of sub-channels.

As it was mentioned before, the spectrum is divided into $N$ parallel sub-channels. Secondary service opportunistically access to the idle sub-channels without imposing interference to the primary users. We model each sub-channel as an ON/OFF source alternating between state ON (busy) and state OFF (idle). Such model for spectrum availability was also 


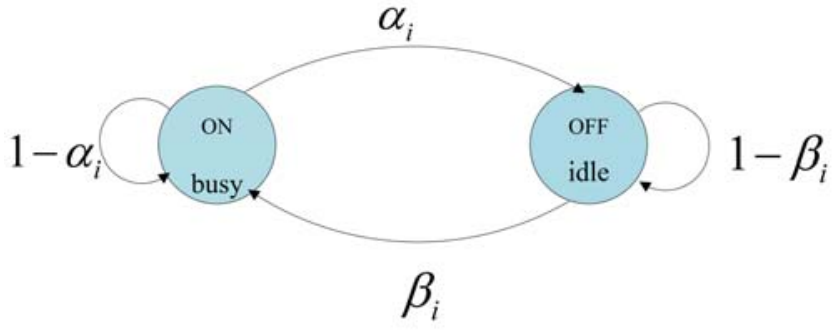

Fig. 2. Two state discrete-time Markov model for the $i$ th sub-channel.

successfully utilized in [9], [11]. Although being very simple, this model is able to capture the temporal characteristics of the channel availability in an OSA system [9], [10].

The secondary service can utilize the OFF sub-channels to make transmission. Let $\alpha_{i}$ be the probability that the $i$ th subchannel transits from state ON to state OFF and $\beta_{i}$ be the probability that the $i$ th sub-channel transits from state OFF to state $\mathrm{ON}$, where $i=1, \ldots, N$. Then, the channel state can be characterized by a two-state Markov chain as shown in Fig. 2. In our treatment of the subject, we also assume that each channel changes its state independently.

The values of $\alpha_{i}$ and $\beta_{i}$, among other things, are a function of the activity of the primary service over sub-channel $i$. In this model, the $i$ th sub-channel is either busy or idle with probability of $p_{B i}$ and $p_{I i}$, respectively; thus

$$
\begin{gathered}
p_{B i}=\left(1-\alpha_{i}\right) p_{B i}+\beta_{i} p_{I i}, \\
p_{I i}=\alpha_{i} p_{B i}+\left(1-\beta_{i}\right) p_{I i} .
\end{gathered}
$$

Probabilities $p_{B i}$ and $p_{I i}$ are then obtained from (1):

$$
p_{B i}=\frac{\beta_{i}}{\alpha_{i}+\beta_{i}}, \quad p_{I i}=\frac{\alpha_{i}}{\alpha_{i}+\beta_{i}} .
$$

In (2), $p_{I i}$ denotes the fraction of time in which the secondary service is able to access the $i$ th sub-channel. The higher the primary service activity in sub-channel $i$ (i.e., higher $\beta_{i}$ and/or lower $\alpha_{i}$ ), the higher $p_{B i}$ is. Therefore in ALOSA scenario we consider $p_{B i}$ as the primary network activity factor.

In practice however, detecting ON and OFF states of a sub-channel is a challenging task which should be conducted by the spectrum sensing mechanism. The spectrum sensing mechanisms are not usually perfectly accurate. In this paper, we develop our analysis based on accurate channel state estimation in Subsection III-A, and then we investigate the impact of inaccurate sub-channel state estimation in Subsection III-B.

2) IL-OSA System: To study the impact of traffic characteristics on the IL-OSA system, we consider a DS-CDMA primary network. In such system, the OFDM secondary service can always access a part of spectrum as far as interference threshold constraint is not violated in the receiver of the primary service.

In DS-CDMA networks, the total received interference caused by other active users in the network coverage area is simply modelled as an additive white Gaussian noise with a power spectral density equal to the product of the background noise power spectral density and a factor $(K-1)$ [15]. The capacity of DS-CDMA network is interference limited [16]. In such networks for each transmitter, a silence period detector is usually utilized to exploit the silence periods by stopping transmission during such periods. This technique reduces the interference and results in a capacity gain. To model the user's activity in DS-CDMA network, users' activity factor, $\nu$, is defined that is the fraction of time in which the user is transmitting (see, e.g., [15], [17]).

Since the secondary user is OFDM, the interference received from the primary service in each $B_{c} \mathrm{~Hz}$ sub-channel can be modelled as an additive white Gaussian noise with power spectral density of $(K-1) N_{0} B_{c}, K \geq 1$. The more activity in the primary network, the higher the value of $K$ is.

Using the results presented in our previous work [17], $K$ can be obtained for the uplink of a DS-CDMA network with chip rate $W$, providing $S$ services each with activity factor $\nu_{s}$, required bit-energy to interference plus noise spectral density, $\rho_{s}$, and bit-rate $R_{s}$ as follows:

$$
K=1+\left(1-(1+f) \sum_{s=1}^{S} n_{s}\left(1+\frac{W}{\rho_{s} \nu_{s} R_{s}}\right)^{-1}\right)^{-1} .
$$

In (3), $f$ is the ratio of the interference originated in the other cells to the interference generated in the home-cell which is a system parameter and is mainly a function of users' spatial distribution in the network coverage area. As it is seen, for a given number of users in each service type (i.e., given $n_{s}$, $s=1, \ldots, S), K$ in (3) is an increasing function of the users' activity. It is also observed in (3) that for a given set of services (i.e., given $\nu_{s}, s=1, \ldots, S$ ), $K$ is an increasing function of the number of users in the network coverage area. In (3), $\left(\nu_{1}, \nu_{2}, \ldots, \nu_{S}\right)$ is an indicator of the users' activity in ILOSA scenario; therefore, we consider $\left(\nu_{1}, \nu_{2}, \ldots, \nu_{S}\right)$ as the primary network activity factor.

\section{AL-OSA: OFDM-OFDM STRUCTURE}

For AL-OSA access, the secondary service should make sub-channels state estimation. This can be conducted by adopting a spectrum sensing technique (for a survey on the spectrum sensing methods see, e.g., [4] and references therein). In ALOSA, secondary service accesses to the corresponding subchannel when the sub-channel is idle. In this case, given an accurate sub-channel state estimation, no interference is caused by the secondary transmitter on the primary receivers. No primary service originated interference is also received in the secondary receiver.

For inaccurate sub-channel state estimation, we have to adopt extra proactive measures to cancel the negative impact of the secondary service transmission on the primary network performance. For instance, one may consider implying the interference threshold constraint to make sure that even in case of miss-detection of a busy sub-channel, the secondary service transmission does not interrupt primary service normal activity. Adopting such measure, of course, decreases the achievable capacity of the secondary service. Therefore, the achievable secondary service capacity is strongly associated with the accuracy of the state estimation. In the following we investigate the impact of the primary network activity for ALOSA scenario with accurate and inaccurate sub-channel state estimation. 


\section{A. Accurate State Estimation}

Given $M$ accessible sub-channels, the maximum achievable capacity of the secondary service $C_{s \mid M}$ is the solution of the following optimization problem.

$$
\begin{gathered}
C_{s \mid M}=\max _{\mathbf{P}_{s}} \sum_{j=1}^{M} \int_{g_{1 j}} p_{I j} B_{c} \log \left(1+\frac{g_{1 j} P_{s j}\left(g_{1 j}\right)}{N_{0} B_{c}}\right) \times \\
d F_{1 j}\left(g_{1 j}\right), \\
\text { s.t. } \sum_{j=1}^{M} \int_{g_{1 j}} P_{s j}\left(g_{1 j}\right) d F_{1 j}\left(g_{1 j}\right) \leq \bar{P}_{s},
\end{gathered}
$$

where $p_{I j}=1-p_{B j}$, (4) is the summation of Shannon (Ergodic) capacity across the accessible sub-channels and $\mathbf{P}_{s}=\left(P_{s 1}\left(g_{11}\right), \ldots, P_{s M}\left(g_{1 M}\right)\right)$. In (4), $P_{s j}$ is only a function of $g_{1 j}$ since transmission is made at the instants when the primary service is inactive. This is because of the fact that during secondary service transmission, the primary service is inactive in sub-channel $j$, thus the imposed interference by the secondary service has no impact on the primary service performance. Therefore, in obtaining $P_{s j}, g_{0 j}$ does not appear in optimization problem (4). In the above optimization problem, (5) implies the secondary service maximum transmission power constraint, $\bar{P}_{s}{ }^{2}$. In the above optimization problem, the interference threshold is out of the picture since the subchannels are idle and not in use by the primary service. Consequently, there is no need to check the interference threshold constraint [7].

The optimization problem in (4) is an instant of Water Filling problem in which the optimal power allocation $\mathbf{P}_{s}^{*}$ can be obtained using Lagrange Multipliers method:

$$
\begin{aligned}
L\left(\mathbf{P}_{s}, \lambda\right)= & \sum_{j=1}^{M} p_{I j} \int_{g_{1 j}} \log \left(1+\frac{g_{1 j} P_{s j}\left(g_{1 j}\right)}{N_{0} B_{c}}\right) d F_{1 j}\left(g_{1 j}\right) \\
& -\lambda\left(\sum_{j=1}^{M} \int_{g_{1 j}} P_{s j}\left(g_{1 j}\right) d F_{1 j}\left(g_{1 j}\right)-\bar{P}_{s}\right) . \text { (6) }
\end{aligned}
$$

Taking the derivative of the Lagrangian in (6) with respect to $\mathbf{P}_{s}$ and letting the derivative equal to zero for $j=1, \ldots, M$, the following is obtained

$$
P_{s j}^{*}\left(g_{1 j}\right)= \begin{cases}\frac{p_{I j}}{\lambda^{*}}-\frac{N_{0} B_{c}}{g_{1 j}} & \text { if } g_{1 j} \geq \frac{\lambda^{*} N_{0} B_{c}}{p_{I j}}, \\ 0 & \text { otherwise. }\end{cases}
$$

In (7), $\lambda^{*}$ is obtained from

$$
\sum_{j=1}^{M} \int_{g_{1 j} \geq \frac{\lambda^{*} N_{0} B_{c}}{p_{I j}}} P_{s j}^{*}\left(g_{1 j}\right) d F_{1 j}\left(g_{1 j}\right)=\bar{P}_{s} .
$$

Eq. (7) indicates that AL-OSA allocates transmission power on sub-channel $j$ in cases where $g_{1 j} \geq \frac{\lambda^{*} N_{0} B_{c}}{p_{I j}}$. As it is seen in (7), by decreasing $p_{I j}$, higher value for $g_{1 j}$ is required so that

\footnotetext{
${ }^{2}$ In some previous studies, e.g., [7], the maximum transmission power constraint is considered as $p_{I j}^{-1} \bar{P}_{s}$ which is increased by decreasing $p_{I j}$, in this paper for brevity we does not imply this assumption.
}

the power is allocated to the corresponding sub-channel. In other words, the range of sub-channel gains for which power is allocated is limited.

Substituting (7) in (4), the maximum achievable capacity, $C_{s \mid M}$, is obtained:

$$
C_{s \mid M}=\sum_{j=1}^{M} p_{I j} B_{c} \int_{g_{1 j} \geq \frac{\lambda^{*} N_{0} B_{c}}{p_{I j}}} \log \left(\frac{p_{I j} g_{1 j}}{\lambda^{*} N_{0} B_{c}}\right) d F_{1 j}\left(g_{1 j}\right) .
$$

The number of accessible sub-channels, $M$, is also a random variable related to the statistics of the primary service activity, thus is a function of the primary network activity factor, $p_{I i}$. Therefore, the total achievable capacity is

$$
C_{s}=E_{M}\left\{C_{s \mid M}\right\}
$$

where $E_{M}\{$.$\} represents expectation according to random$ variable $M$.

\section{B. Inaccurate State Estimation}

In practice, spectrum sensing mechanisms are not perfect. Let $\delta_{i}$, be the probability of miss-detection, i.e., the probability of estimating the state of sub-channel $i$ as idle (OFF) while it is busy $(\mathrm{ON})$, and $\epsilon_{i}$ be the probability of false-alarm, i.e., the probability of estimating the channel state as busy (ON) while it is idle (OFF).

Parameters $\delta_{i}$ and $\epsilon_{i}$ are specified by the receiver operating characteristic (ROC) curve which gives the probability of detection, $1-\delta_{i}$, as a function of $\epsilon_{i}$. ROC is a system characteristics of an adopted spectrum sensing technique [4]. For a given value of $\delta_{i}, \epsilon_{i}$ is obtained using the ROC curve.

Inaccurate spectrum sharing results in collision between the primary and the secondary service transmissions. Therefore, if the spectrum sharing system is not designed to manage the miss-detection incidents, the collision between the primary and the secondary service transmissions can potentially reduce the actual capacity of the primary network. However, in practice some sort of tolerability to collision is considered for the primary network. In other words, the spectrum sensing mechanism is utilized so that its probability of miss-detection satisfies the collision probability constraint of the primary service.

If $\xi$ is the maximum allowable probability of collision, then the probability of detection in the sub-channel $j, 1-\delta_{j}$, is obtained so that

$$
\delta_{j} \leq \operatorname{Pr}\left\{P_{s j} g_{0 j}>Q\right\}=\xi .
$$

Based on the above approach, the impact of inaccurate spectrum sensing on the capacity of the primary network is already taken into account by tuning the parameters of the spectrum sensing technique.

In case of inaccurate spectrum sensing, the fraction of time that the secondary service is able to access to the sub-channel $j, \hat{p}_{I j}$, is

$$
\hat{p}_{I j}=\left[p_{I j}\left(1-\epsilon_{j}\right)+p_{B j} \delta_{j}\right] .
$$

Therefore, the optimization problem in (4) can be approximated as 


$$
\begin{gathered}
C_{s \mid M}=\max _{\mathbf{P}_{s}} \sum_{j=1}^{M} \int_{g_{1 j}} \hat{p}_{I j} B_{c} \log \left(1+\frac{g_{1 j} P_{s j}\left(g_{1 j}\right)}{N_{0} B_{c}}\right) \times \\
d F_{1 j}\left(g_{1 j}\right) \\
\text { s.t. } \sum_{j=1}^{M} \int_{g_{1 j}} P_{s j}\left(g_{1 j}\right) d F_{1 j}\left(g_{1 j}\right) \leq \bar{P}_{s}
\end{gathered}
$$

Note that in the case of miss-detection, an interference term appears in (13) that should be added to $N_{0} B$ in the logarithm. The interference term can be ignored because of the following. In most cases a signalling protocol in the form of request-tosend/clear-to-send (RTS/CTS) is employed by the secondary service [4]. In the case of miss-detection, the level of received interference from the primary network is very low. Therefore, both secondary transmitter and secondary receiver detect an idle sub-channel. As a result, the corresponding interference term can be ignored in (14).

In the above, the interference threshold constraint does not appear. It is due to the fact that it is already considered through (11). In other words, in case of miss-detection, the interference threshold constraint may be violated. However, the spectrum sensing parameters have been already designed based on (11) so that the violation probability is always less than or equal to $\xi$. Replacing $p_{I j}$ by $\hat{p}_{I j}$ in (7) the optimal transmission power of the secondary service is obtained.

\section{IL-OSA: CDMA-OFDM STRUCTURE}

In IL-OSA, secondary service can always make access to a part of spectrum as far as the interference threshold constraint is not violated in the receiver of the primary service. Assume that there are $M$ accessible sub-channels for the secondary service. The maximum achievable capacity of the secondary service over $M$ accessible sub-channels is the solution of the following optimization problem:

$$
\begin{array}{r}
C_{s \mid M}=\max _{\mathbf{P}_{s}} \sum_{j=1}^{M} B_{c} \int_{g_{0 j}, g_{1 j}} \log \left(1+\frac{g_{1 j} P_{s j}\left(g_{0 j}, g_{1 j}\right)}{K N_{0} B_{c}}\right) \times \\
d F_{0 j}\left(g_{0 j}\right) d F_{1 j}\left(g_{1 j}\right) \quad(15) \\
\text { s.t. } \sum_{j=1}^{M} \int_{g_{0 j}, g_{1 j}} P_{s j}\left(g_{0 j}, g_{1 j}\right) d F_{0 j}\left(g_{0 j}\right) d F_{1 j}\left(g_{1 j}\right) \leq \bar{P}_{s},(16) \\
\sum_{j=1}^{M} \int_{g_{0 j}, g_{1 j}} g_{0 j} P_{s j}\left(g_{0 j}, g_{1 j}\right) d F_{0 j}\left(g_{0 j}\right) d F_{1 j}\left(g_{1 j}\right) \leq G Q .
\end{array}
$$

In the above $\mathbf{P}_{s}=\left(P_{s 1}\left(g_{01}, g_{11}\right), \ldots, P_{s M}\left(g_{0 M}, g_{1 M}\right)\right)$, (16) is the average transmission power constraint for the secondary service, $G$ is the processing gain of DS-CDMA primary network and $Q$ is the interference threshold. Constraint (17) implies the primary service's interference threshold in which secondary service's operation in each sub-channel acts as a narrow-band interference that its energy is spread over the whole bandwidth in the DS-CDMA receiver; thus, being multiplied by $1 / G$. Here, $P_{s j}$ is a function of both $g_{0 j}$ and $g_{1 j}$ since transmission is made at the instants when the primary service is also active. Therefore, the secondary service transmission power should be obtained so that the impact on the primary service performance (which is related to $g_{0 j}$ ) is kept within an acceptable interval based on (17).

If the activity factor in the primary network varies over time, it results in varying $K$. To exploit varying primary network activity, one may consider adaptive adjustment of $Q$. For higher activity in the primary network, $K$ is increased; thus, $Q$ should be decreased. For lower activity in the primary network, $K$ is decreased; therefore, $Q$ could be increased.

The optimization problem in (15) can also be considered as a modified version of the classic Water Filling problem. The optimal power allocation $\mathbf{P}_{s}^{*}$ is then obtained using Lagrange Multipliers method:

$$
\begin{aligned}
& L\left(\mathbf{P}_{s}, \lambda_{1}, \lambda_{2}\right)= \\
& \sum_{j=1}^{M} \int_{g_{0 j}, g_{1 j}} \log \left(1+\frac{g_{1 j} P_{s j}\left(g_{0 j}, g_{1 j}\right)}{K N_{0} B_{c}}\right) d F_{0 j}\left(g_{0 j}\right) d F_{1 j}\left(g_{1 j}\right)- \\
& \lambda_{1}\left(\sum_{j=1}^{M} \int_{g_{0 j}, g_{1 j}} P_{s j}\left(g_{0 j}, g_{1 j}\right) d F_{0 j}\left(g_{0 j}\right) d F_{1 j}\left(g_{1 j}\right)-\bar{P}_{s}\right)- \\
& \lambda_{2}\left(\sum_{j=1}^{M} \int_{g_{0 j}, g_{1 j}} g_{0 j} P_{s j}\left(g_{0 j}, g_{1 j}\right) d F_{0 j}\left(g_{0 j}\right) d F_{1 j}\left(g_{1 j}\right)-G Q\right) .
\end{aligned}
$$

Taking the derivative of $L\left(\mathbf{P}_{s}, \lambda_{1}, \lambda_{2}\right)$ with respect to $\mathbf{P}_{s}$ and letting the result equal to zero, we obtain

$$
\frac{g_{1 j}}{K N_{0} B_{c}+g_{1 j} P_{s j}^{*}}=\lambda_{1}^{*}+\lambda_{2}^{*} g_{0 j}, \quad j=1,2, \ldots, M .
$$

Thus, the optimal power allocation, $\mathbf{P}_{s}^{*}$, is

$$
P_{s j}^{*}\left(g_{0 j}, g_{1 j}\right)= \begin{cases}\frac{1}{\lambda_{1}^{*}+\lambda_{2}^{*} g_{0 j}}-\frac{K N_{0} B_{c}}{g_{1 j}} & \text { if }\left(g_{0 j}, g_{1 j}\right) \in \Theta, \\ 0 & \text { otherwise, }\end{cases}
$$

where $\Theta=\left\{\left(g_{0 j}, g_{1 j}\right) \mid \frac{g_{1 j}}{\lambda_{1}^{*}+\lambda_{2}^{*} g_{0 j}} \geq K N_{0} B_{c}\right\}$, and $\lambda_{1}^{*}, \lambda_{2}^{*}$ are obtained from the following set of equations:

$$
\begin{gathered}
\sum_{j=1}^{M} \int_{\frac{g_{1 j}}{\lambda_{1}^{*}+\lambda_{2}^{*} g_{0 j}} \geq K N_{0} B}\left(\frac{1}{\lambda_{1}^{*}+\lambda_{2}^{*} g_{0 j}}-\frac{K N_{0} B_{c}}{g_{1 j}}\right) \times \\
d F_{0 j}\left(g_{0 j}\right) d F_{1 j}\left(g_{1 j}\right)=\bar{P}_{s}, \\
\sum_{j=1}^{M} \int_{\frac{g_{1 j}}{\lambda_{1}^{*}+\lambda_{2}^{*} g_{0 j}} \geq K N_{0} B}\left(\frac{g_{0 j}}{\lambda_{1}^{*}+\lambda_{2}^{*} g_{0 j}}-\frac{g_{0 j}}{g_{1 j}} K N_{0} B_{c}\right) \times \\
d F_{0 j}\left(g_{0 j}\right) d F_{1 j}\left(g_{1 j}\right)=G Q .
\end{gathered}
$$

Using (19) and (15), $C_{s \mid M}$ is obtained as

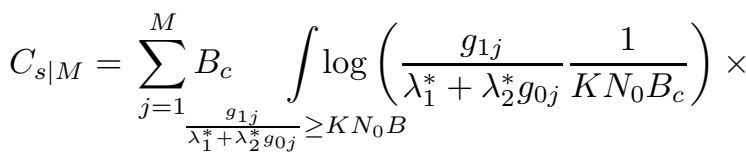

$$
\begin{aligned}
& d F_{0 j}\left(g_{0 j}\right) d F_{1 j}\left(g_{1 j}\right),
\end{aligned}
$$




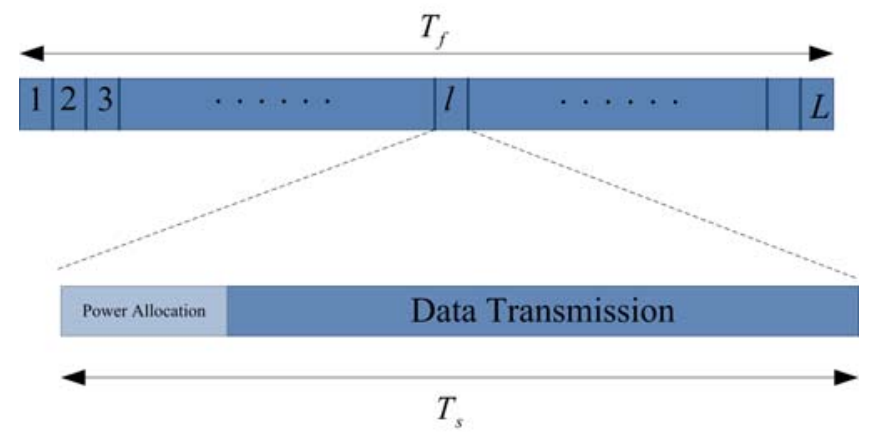

Fig. 3. Data structure for sub-channel $j$.

which can be written as follows:

$$
C_{s \mid M}=\sum_{j=1}^{M} B_{c} \int_{0}^{\infty} \log \left(\frac{y_{j}}{K N_{0} B_{c}}\right) f_{Y}\left(y_{j}\right) d y_{j},
$$

where

$$
y_{j}=\frac{g_{1 j}}{\lambda_{1}^{*}+\lambda_{2}^{*} g_{0 j}}, j=1,2, \ldots, M,
$$

is a random variable. For Rayleigh fading the pdf of $y_{j}$ is (see Appendix A):

$$
f_{Y}\left(y_{j}\right)=e^{-\lambda_{1}^{*} y_{j}}\left(\frac{\lambda_{1}^{*}}{1+\lambda_{2}^{*} y_{j}}+\frac{\lambda_{2}^{*}}{\left(1+\lambda_{2}^{*} y_{j}\right)^{2}}\right) .
$$

\section{IMPACT OF CHANNEL GAIN}

In the derivations of the maximum achievable capacity for the AL-OSA and the IL-OSA scenarios based on the optimization problems in (4) and (15) we assume that the secondary and the primary channels only have fading effect. However, in practice the channel gains also include shadowing effect as well as the distance dependent path-loss effect. Since the distance dependent path-loss and shadowing are implied in the larger time scales, in this paper to solve the optimization problems in (4) and (15) we propose a multiple time-scale approach as described bellow.

In our treatment of the subject, time is divided into frames, indexed by $n$, each with $T_{f}$ seconds duration. Frame duration $T_{f}$ is small enough comparing to the shadowing coherence time so that during each frame, the shadowing gain is almost constant. For usual mobility profiles, it is also reasonable to assume that the distance dependent path-loss between the transmitter and the receiver remains constant in time scale $T_{f}$. Each frame consists of $L$ slots indexed by $l, l=1,2, \ldots, L$, each with $T_{s}$ seconds duration (Fig. 3). The duration of timeslots $T_{s}$ is small enough comparing to the multi-path fading coherence time, so that during each time-slot the fading gain is almost constant. The transmission power of the secondary service user at time-slot $l$ in frame $n$ of sub-channel $j$ is denoted by $P_{s j}(n, l)$.

Therefore, the actual secondary channel gain at time-slot $l$ of frame $n$ in sub-channel $j$ denoted by $a_{1 j}(n, l)$ is [18]:

$$
a_{1 j}(n, l)=\kappa_{1} d_{1}(n)^{-\gamma} 10^{\frac{\xi_{1}(n)}{10}} g_{1 j}(n, l),
$$

where $\gamma$ is the path-loss exponent, $d_{1}(n)$ is the distance between the secondary transmitter and receiver at frame $n, \kappa_{1}$ is an environment dependent parameter and $g_{1 j}(n, l)$ is unitmean channel fading ${ }^{3}$. In (25), shadowing is modelled as a Log-Normal random variable with standard deviation $\sigma_{1(\mathrm{~dB})}$. Therefore, in the corresponding shadowing term of (25), i.e., $10^{\frac{\xi_{1}(n)}{10}}, \xi_{1}$ is a Normal random variable with zero mean and variance $\sigma_{1(\mathrm{~dB})}^{2}$. In (25) it is assumed that shadowing is the same for all sub-channels.

We use the same model for the channel between the secondary transmitter and the primary receiver which consists of a distance dependent path-loss with an exponent $\gamma$, a Log-Normal shadowing with standard deviation $\sigma_{0(d B)}$, and a unit-mean channel fading $g_{0 j}(n, l)$ as following.

$$
a_{0 j}(n, l)=\kappa_{0} d_{0}(n)^{-\gamma} 10^{\frac{\xi_{0}(n)}{10}} g_{0 j}(n, l),
$$

where $d_{0}(n)$ is the distance between the secondary transmitter and the primary receiver and $\xi_{0}$ is defined same as $\xi_{1}$ as a Normal random variable with zero mean and variance $\sigma_{0(\mathrm{~dB})}^{2}$. Shadowing is not frequency selective and is the same for all sub-channels.

Considering the channel models in (25) and (26), the optimization problems in (4) and (15) should be modified by replacing $g_{0 j}$ and $g_{1 j}$, with $a_{0 j}$ and $a_{1 j}$, respectively. In multiple time-scale scenario, the channel gains in problems (4) and (15) are measured through the following steps: (i) the values of the path-loss and shadowing are measured in time scale $T_{f}$. These values remain constant during the corresponding frame $n$, (ii) within each frame $n$, for each time-slot $l, l=1, \ldots, L$, the problems in (4) and (15) are solved based on $g_{0 j}(n, l)$ and $g_{1 j}(n, l)$ measured at time scale $T_{s}$.

Here, we obtain the achievable capacity of the AL-OSA and IL-OSA systems. For brevity of the expositions, we assume that one sub-channel is involved in the spectrum sharing; therefore, the total secondary service transmission power is allocated for transmission on the selected subchannel. The sub-channel is selected by the regulator or primary service operator. Following the same line of argument as in Sections III and IV extending the results in the following sub-section to the case with more than one sub-channel is straightforward.

\section{A. Achievable Capacity for AL-OSA}

Using the channel models in (25) and (26) the optimization problem in (4) for a given sub-channel $j$ is converted to

$$
\begin{aligned}
C_{s j}=\max _{P_{s j}(n, l)} & \mathbb{E}_{a_{1 j}}\left[p_{I j} B_{c} \log \left(1+\frac{a_{1 j}(n, l) P_{s j}(n, l)}{N_{0} B_{c}}\right)\right], \\
\text { s.t. } & \mathbb{E}_{a_{1 j}}\left[P_{s j}(n, l)\right] \leq \bar{P}_{s},
\end{aligned}
$$

where $\mathbb{E}_{x}\{h(x)\} \triangleq \int_{x} h(x) f_{X}(x) d x$, and $f_{X}(x)$ is the pdf of random variable $X$.

The above optimization problem is solved at the beginning of each time-slot. Following the same techniques as in Section

\footnotetext{
${ }^{3}$ Using the channel model in (25), the assumption of unit-mean fading we made in Section II can be justified by considering a proper corrective coefficient in $\kappa_{1}$.
} 
III,

$$
P_{s j}^{*}(n, l)= \begin{cases}\frac{p_{I j}}{\lambda^{*}}-\frac{N_{0} B_{c}}{a_{1 j}(n, l)} & \text { if } a_{1 j}(n, l) \geq \frac{\lambda^{*} N_{0} B_{c}}{p_{I j}} \\ 0 & \text { otherwise, }\end{cases}
$$

where $\lambda^{*}$ is obtained from

$$
\mathbb{E}_{a_{1 j}}\left(\frac{p_{I j}}{\lambda^{*}}-\frac{N_{0} B_{c}}{a_{1 j}(n, l)}\right)^{+}=\bar{P}_{s}
$$

where $(x)^{+} \triangleq \max \{0, x\}$. Using (25), the allocated power $P_{s j}^{*}(n, l)$ can be re-written as

$$
P_{s j}^{*}(n, l)= \begin{cases}\frac{p_{I j}}{\lambda^{*}}-\frac{\mathcal{N}_{1}(n) B_{c}}{g_{1 j}(n, l)} & \text { if } g_{1 j}(n, l) \geq \frac{\lambda^{*} \mathcal{N}_{1}(n) B_{c}}{p_{I j}} \\ 0 & \text { otherwise, }\end{cases}
$$

where

$$
\mathcal{N}_{1}(n) \triangleq \frac{N_{0}}{\kappa_{1}} d_{1}(n)^{\gamma} 10^{\frac{-\xi_{1}(n)}{10}} .
$$

At the beginning of each frame $n, \mathcal{N}_{1}(n)$ is obtained using (28); consequently the optimal allocated power of the secondary service on sub-channel $j$ is obtained from (27).

\section{B. Achievable Capacity for IL-OSA}

Using the same method as it was used in Subsection V-A for a given sub-channel $j$, the optimization problem in (15) is converted to

$$
\begin{aligned}
C_{s j}=\max _{P_{s j}(n, l)} & \mathbb{E}_{a_{1 j}} \mathbb{E}_{a_{0 j}}\left[B_{c} \log \left(1+\frac{a_{1 j}(n, l) P_{s j}(n, l)}{K N_{0} B_{c}}\right)\right], \\
\text { s.t. } & \mathbb{E}_{a_{1 j}} \mathbb{E}_{a_{0 j}}\left[P_{s j}(n, l)\right] \leq \bar{P}_{s}, \\
& \mathbb{E}_{a_{1 j}} \mathbb{E}_{a_{0 j}}\left[a_{0 j} P_{s j}(n, l)\right] \leq G Q .
\end{aligned}
$$

Note that in practice $Q$ can be also evaluated per frame.

Following the same steps similar to those in Section IV, the optimal transmitted power is then obtained as follows:

$$
P_{s j}^{*}(n, l)=\left(\frac{1}{\lambda_{1}^{*}+\lambda_{2}^{*} a_{0 j}(n, l)}-\frac{K N_{0} B_{c}}{a_{1 j}(n, l)}\right)^{+},
$$

where $\lambda_{1}^{*}$ and $\lambda_{2}^{*}$ are obtained from

$$
\mathbb{E}_{a_{1 j}} \mathbb{E}_{a_{0 j}}\left(\frac{1}{\lambda_{1}^{*}+\lambda_{2}^{*} a_{0 j}(n, l)}-\frac{K N_{0} B_{c}}{a_{1 j}(n, l)}\right)^{+}=\bar{P}_{s},
$$

and

$\mathbb{E}_{a_{1 j}} \mathbb{E}_{a_{0 j}}\left(\frac{a_{0 j}(n, l)}{\lambda_{1}^{*}+\lambda_{2}^{*} a_{0 j}(n, l)}-\frac{a_{0 j}(n, l)}{a_{1 j}(n, l)} K N_{0} B_{c}\right)^{+}=G Q$.

Using the channel models in (25) and (26), the allocated power $P_{s j}^{*}(n, l)$ is then obtained as

$$
P_{s j}^{*}(n, l)=\left(\frac{1}{\lambda_{1}^{*}+\tilde{\lambda}_{2}^{*}(n) g_{0 j}(n, l)}-\frac{K \mathcal{N}_{1}(n) B_{c}}{g_{1 j}(n, l)}\right)^{+},
$$

where

$$
\tilde{\lambda}_{2}^{*}(n)=\kappa_{0} d_{0}(n)^{-\gamma} 10^{\frac{\xi_{0}(n)}{10}} \lambda_{2}^{*},
$$

and

$$
\mathcal{N}_{1}(n)=\frac{N_{0}}{\kappa_{1}} d_{1}(n)^{\gamma} 10^{\frac{-\xi_{1}(n)}{10}} .
$$

At the beginning of each frame $n, \mathcal{N}_{1}(n)$ and $\tilde{\lambda}_{2}^{*}(n)$ are calculated from (30) and (31), respectively; then the optimal allocated power of the secondary service on sub-channel $j$ is obtained from (29).
TABLE I

SiMUlation PARAMETERS

\begin{tabular}{l|c}
\hline Parameter & Value \\
\hline Spectrum Bandwidth $(B)$ & $3.84 \mathrm{MHz}$ \\
Propagation loss exponent & 4 \\
Cell radius & $1000 \mathrm{~m}$ \\
$d_{0}=d_{1}$ & $100 \mathrm{~m}$ \\
Standard dev. of Log-Normal shadowing & $8 \mathrm{~dB}$ \\
Shadowing Coherence Time & $150 \mathrm{~ms}$ \\
Fading Coherence Time & $3 \mathrm{~ms}$ \\
$L$ & 50 \\
$N$ & 64 \\
$B_{c}$ & $60 \mathrm{KHz}$ \\
$\kappa_{0}=\kappa_{1}$ & 1 \\
Background noise power spectral density $\left(N_{0}\right)$ & $-174.0 \mathrm{dBm} / \mathrm{Hz}$ \\
$\bar{P}_{s}$ & $1 \mathrm{Watt}$ \\
$G$ & 125 \\
$Q$ & $N_{0} B$ \\
$f$ & 0.5 \\
Minimum required $E_{b} / I_{0}$ & $2 \mathrm{~dB}$ \\
\hline
\end{tabular}

\section{Simulations}

In this section we evaluate the performance of both ALOSA and IL-OSA systems through simulations. In our simulations we adopted Monte-Carlo method with sufficient number of iterations. The sub-channel gains are updates in each iteration based on the channel model presented in Table I. The main performance measure in this section is the normalized maximum achievable capacity, $\frac{C_{s}}{M B_{c}}$. This measure indicates the efficiency of each scenario in sharing spectrum. We then study the impact of adopting different spectrum sharing scenarios and primary network activity.

Assuming users with uniform spatial distribution in the primary network coverage area with i.i.d. channel gains, Markov model for all sub-channels are also identical. Note that in our simulations perfect CSI availability is assumed thus, the results presented in this section should be treated as an actual upper-bound for practical cases with no CSI or partial CSI.

1) AL-OSA System: To show the impact of primary network activity on the the normalized maximum achievable capacity, we simulate an OFDM-OFDM system with 64 i.i.d. subchannels where $p_{B i}=p_{B}, \delta_{i}=\delta, i=1, \ldots, 64$. In Fig. 4, the normalized maximum achievable capacity, $C_{s} / M B_{c}$ is plotted versus the primary network activity factor, $p_{B}$ for different values of miss-detection probability, $\delta$. Since in practice, the value of miss-detection probability is usually less than 0.1 [9], [19], we perform our simulations for $\delta=0,0.05,0.1$. As it is observed, the normalized maximum achievable capacity is significantly decreased by increasing the primary network activity factor. It is also seen that the decrease in normalized maximum achievable capacity is almost linear with the increase of $p_{B}$. Furthermore, the slope of decrease is reduces by increasing $\delta$.

2) IL-OSA System: To show the impact of the primary network activity factor on the total achievable capacity, we simulate a CDMA-OFDM system. The primary DS-CDMA network serves 50 active primary users and $\nu$ is the primary network activity factor. In our simulations, for each value of $M$, the secondary service selects the first $M$ channels sorted 


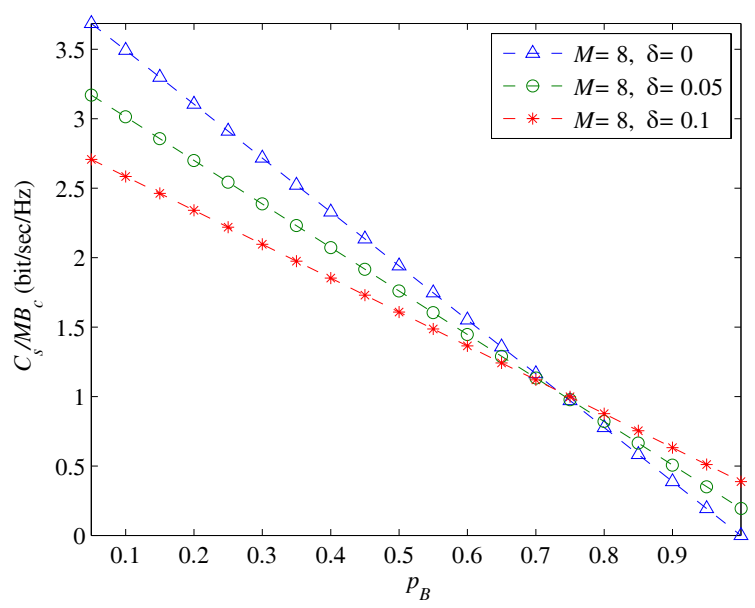

Fig. 4. Normalized maximum achievable capacity of the secondary user, $C_{s} / M B_{c}$, in AL-OSA system $v s$. the primary network activity factor, $p_{B}$ $\left(p_{B i}=p_{B}, \delta_{i}=\delta\right)$, for $\delta=0,0.05,0.1$.

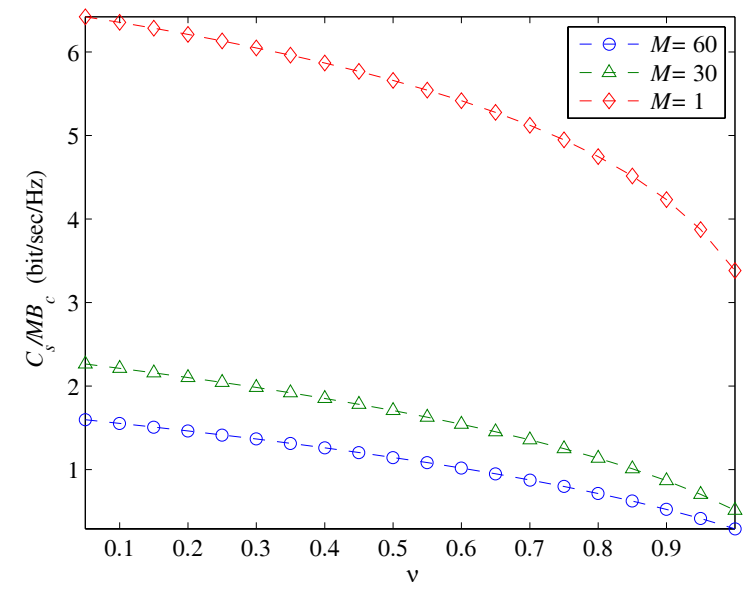

Fig. 5. Normalized maximum achievable capacity of the secondary user, $C_{s} / B_{c}$, in IL-OSA versus the primary network activity factor, $\nu$, for $M=1$, $M=30, M=60$.

based on $g_{1 i} / g_{0 i}$ in descending order ${ }^{4}$.

First we study the impact of the number of accessible sub-channels. Fig. 5 demonstrates the normalized maximum achievable capacity, $C_{s} / M B_{c}$ versus $\nu$, for $M=1,30,60$. It is observed that for different values of $M, C_{s} / M B_{c}$ is significantly decreased by increasing the primary network activity factor. Note that since $\bar{P}_{s}$ is fixed, the spectral efficiency, $C_{s} / M B_{c}$, is increased when $M$ is decreased.

We also study the impact of $N$, on $C_{s} / M B_{c}$. Fig. 6 shows $C_{s} / M B_{c}, M=1$ for the DC-CDMA primary network with 50 active users, versus the primary network activity factor, $\nu$. Here we also assume that the secondary service selects the sub-channel with maximum $g_{1 i} / g_{0 i}$. As it is observed, by increasing $N$, the normalized maximum achievable capacity is increased. This is mainly because the probability of finding

\footnotetext{
${ }^{4}$ Note that in IL-OSA, the best sub-channel can be considered as the one which achieves the maximum capacity, and imposes the minimum interference at the same time, i.e., $\max _{i} g_{1 i} / g_{0 i}$.
}

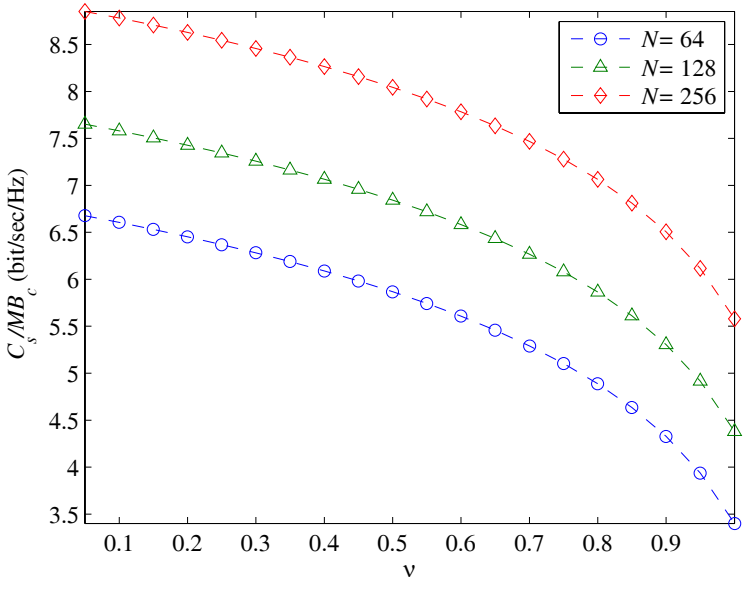

Fig. 6. Normalized maximum achievable capacity of the secondary user, $C_{s} / B_{c}$, in IL-OSA versus the primary network activity factor, $\nu$, for $M=1$ and $N=64, N=128, N=256$

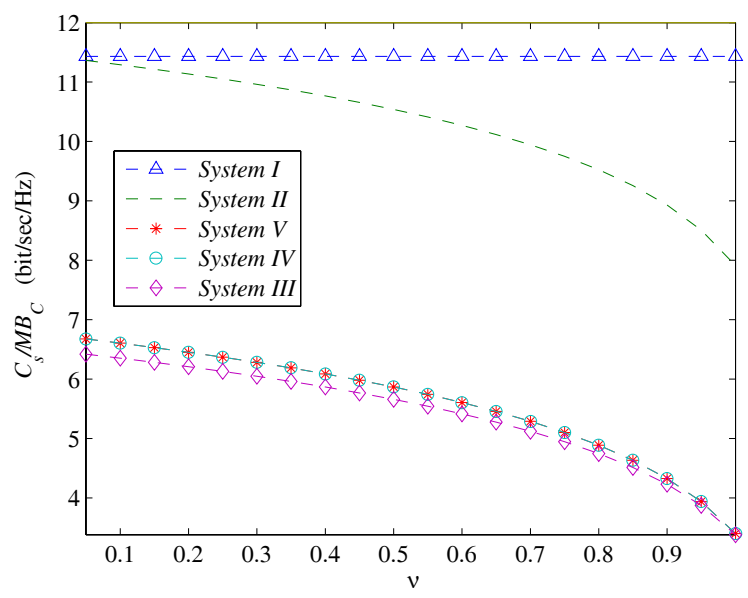

Fig. 7. Normalized maximum achievable capacity of Systems I-V.

a sub-channel with a larger value of $g_{1 i} / g_{0 i}$ is increased by increasing $N$.

We then compare $C_{s} / M B_{c}$ with what has been obtained in the previous studies to show the impact of the primary network activity factor as well as the secondary service transmit power constraint, $\bar{P}_{s}$. In Fig. 7, $C_{s} / M B_{c}$ is plotted versus $\nu$ for $M=1$ when different values of $Q$ and $\bar{P}_{s}$ are considered. We then simulate the following five systems. System I is an ILOSA system based on the model presented in [8], in which $P_{s}$ is not considered as a system constraint, and a fixed primary network activity is also assumed. This assumption results in a fixed $K=1$. We also set $Q=N_{0} B$. System II is also an ILOSA system modelled based on a modified version of the one in [8], in which the primary network activity is also included in the model through $K$ based on (3). System II does not have transmit power constraint, we also set $Q=N_{0} B$. In System III, both transmit power constraint and the primary network activity are included. For System III we set $\bar{P}_{s}=1 \mathrm{~W}$, and $Q=N_{0} B$. System IV and System V are similar to System III with $Q=10 N_{0} B$ and $Q=100 N_{0} B$, respectively. 
The gap between System I and System II in Fig. 7 is due to the impact of the primary system activity. The gap is then widened between System I and System III because of including the secondary system transmit power constraint. The results presented in Fig. 7 also indicates that in spite of the availability of radio resource in the primary network for spectrum sharing, i.e., increasing $Q$, the secondary service power constraint does not allow further improving of the achievable capacity.

3) Comparing AL-OSA and IL-OSA: To compare AL-OSA and IL-OSA in a fair manner, we consider two following systems. In both cases we set $M=1$ and assume that the secondary service has enough backlogged traffic to be transmitted upon availability of the radio resource.

The simulated AL-OSA is a system with network activity parameter, $p_{B}$. On average, for this system $p_{I}=1-p_{B}$ is the fraction of the whole bandwidth which can be utilized by the secondary service. Simulations are conducted for $M=1$ and the best sub-channel with $\max _{i} g_{1 i}$ is assumed to be selected among all accessible sub-channels. The total secondary service transmit power is then allocated to the selected sub-channel based on the optimization problem in (4). Since in AL-OSA the selected sub-channel is not in use by the primary network, the secondary transmission does not affect the primary network.

In IL-OSA, equivalent to the above setting, we assume that the secondary service is eligible to allocate a fraction of $p_{I}$ from the whole available radio resource over the best available sub-channel, i.e., $\left(1-p_{B}\right) G Q$. Since comparing to AL-OSA, the secondary service activity in IL-OSA interferes the primary network, corresponding to the above mentioned AL-OSA scenario, the best sub-channel is assumed to be the one with the maximum $g_{1 i} / g_{0 i}$. This selection policy tries to achieve a higher secondary service capacity, while implies a lower value of interference on the primary network. Power allocation is then conducted based on the optimization problem in (15).

In Fig. 8 the normalized maximum achievable capacity, $C_{s} / M B_{c}$, is plotted versus $p_{B}$ for aforementioned AL-OSA and IL-OSA systems. For lower values of $p_{B}$, on average, ALOSA can access to a larger of sub-channels, and it only needs to verify the transmit power constraint. Therefore, comparing to the case of IL-OSA which should also apply the interference threshold, it is more probable for AL-OSA to find subchannel(s) with very high achievable capacity. As a result, we expect higher achievable capacity for AL-OSA in cases of low values of $p_{B}$, this is confirmed by the results in Fig. 8 .

Comparing to the case of IL-OSA which can track the whole bandwidth, it is more probable for IL-OSA to find subchannel(s) with very high achievable capacity. This is because of the fact that for higher values of $p_{B}$, on average, ALOSA may access to a smaller number of sub-channels, and yet it must implement the secondary service transmit power constraint. Therefore, we expect higher achievable capacity for IL-OSA in cases of high values of $p_{B}$. This is also confirmed by the results in Fig. 8 .

According to the above results, we can conclude that in our simulation scenario, AL-OSA outperforms IL-OSA for lower values of $p_{B}$. However, for higher values of $p_{B}$, IL-

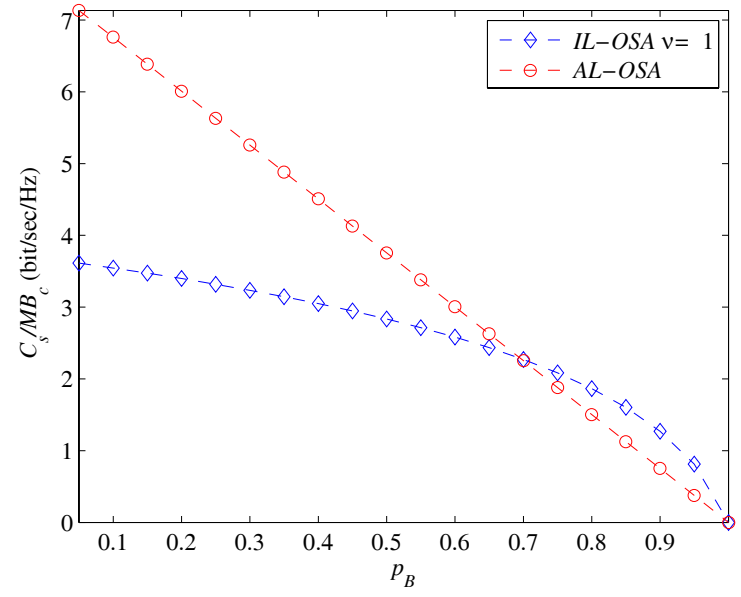

Fig. 8. Normalized maximum achievable capacity for AL-OSA and IL-OSA, versus $p_{B}$, for $M=1$.

OSA performs better than that of AL-OSA.

\section{Conclusions and Practical Considerations}

The main objective of this paper was to formulate the impact of the primary service activity on the achievable capacity of the secondary service. We considered two OSA scenarios: 1) Access-Time Limited OSA (AL-OSA) in which the main constraint for the secondary service is the access time, and 2) Interference Limited OSA (IL-OSA) where the interference level in the receiver of the primary user is the main constraint for the secondary service transmitter.

In AL-OSA we modelled the channel availability through an ON/OFF Markov model. The parameters of this model are determined based on the primary network activity. The systems in which both primary and secondary services make use of OFDM is an example of AL-OSA. We then obtained the maximum achievable capacity for AL-OSA based on primary network activity factor, which is modelled as the probability of a sub-channel to be in the busy state. Our analytical results showed that the maximum achievable capacity is significantly increased through decreasing the primary network activity factor. This result was also confirmed by simulations. Since we assume both perfect CSI availability and perfect channel state estimation, the result we obtained for achievable capacity actually constitutes an upper-bound for practical cases.

In practice, approaching the upper-bound can be achieved by employing channel accessibility estimation methods with high level of accuracy. For CSI estimation, different approaches can also be employed including considering a band coordinator or adopting a blind estimation technique for channel estimation. However, the former idea results in structure complexity and the latter in computational complexity. We also study the impact of inaccurate channel estimation and concluded that it may result in transmission collision and thus may degrade the quality of service in the primary network.

In IL-OSA the primary network activity reflects itself in the interference level. The more active the primary service, the higher the level of interference is. Formulating this effect in the optimization problem for achievable capacity, we obtained 
the achievable capacity for IL-OSA. The IL-OSA scenario is an appropriate model for the case that the primary service makes use of DS-CDMA and the secondary service makes use of OFDM. We then obtained the maximum achievable capacity for IL-OSA based on primary network activity factor which is defined as the average fraction of time a user is transmitting. Our analytical results showed that the maximum achievable capacity is also significantly increased by decreasing the primary network activity factor. This result was confirmed by simulations as well. Since we assumed perfect CSI availability, the result obtained for achievable capacity constitutes an upper-bound for practical cases.

If the secondary user is able to adaptively select $M$ out of $N$ sub-channels in an IL-OSA system, it would add a new degree of freedom for the secondary service. An idea could be making the selection with the objective of maximizing the achievable capacity and at the same time, minimizing the negative impact on the primary network.

Since in practice both CDMA and most of the OFDM cellular systems are single frequency, the operation of the secondary service in the primary band may impose unexpected interference on the receivers in the adjacent cells. To deal with this issue, one may add new constraints to the optimization problems in (4) and (15) for AL-OSA and IL-OSA systems, respectively or consider a conservative value for $Q$.

Particularly, a combination of AL-OSA and IL-OSA can be considered. In such combination AL-OSA access the spectrum while it is idle; during busy periods IL-OSA is adopted.

\section{APPENDIX A}

Here we obtain the pdf of random variable $y_{j}$ in (23). The probability distribution function of random variable $y_{j}$ is

$$
F_{Y}\left(y_{j}\right)=\operatorname{Pr}\left\{Y \leq y_{j}\right\}=\operatorname{Pr}\left\{g_{1 j} \leq y_{j}\left(\lambda_{1}^{*}+\lambda_{2}^{*} g_{0 j}\right)\right\} \text {. }
$$

Since we assume that the channel fading is Rayleigh, both $g_{0 j}$ and $g_{1 j}$ (which are power gains) are i.i.d. unit-mean random variables with exponential distribution; therefore, the probability distribution function of $y_{j}$ is

$$
F_{Y}\left(y_{j}\right)=\int_{0}^{\infty} \int_{0}^{\left(\lambda_{1}^{*}+\lambda_{2}^{*} g_{0 j}\right) y_{j}} e^{-g_{0 j}} e^{-g_{1 j}} d g_{0 j} d g_{1 j} .
$$

The pdf is then obtained by taking the derivative of (33) with respect to $y_{j}$ :

$$
f_{Y}\left(y_{j}\right)=\int_{0}^{\infty}\left(\lambda_{1}^{*}+\lambda_{2}^{*} g_{0 j}\right) e^{-g_{0 j}} e^{-\left(\lambda_{1}^{*}+\lambda_{2}^{*} g_{0 j}\right) y_{j}} d g_{0 j} .
$$

Utilizing integration by part in (34) yields

$$
\begin{aligned}
& \left.f_{Y}\left(y_{j}\right)=-\lambda_{1}^{*} e^{-\lambda_{1}^{*}} \frac{1}{1+\lambda_{2}^{*} y_{j}} e^{-\left(1+\lambda_{2}^{*} y_{j}\right) g_{0 j}}\right]_{0}^{\infty}- \\
& \left.\lambda_{2}^{*} e^{-\lambda_{1}^{*}}\left(\frac{g_{0 j}}{1+\lambda_{2}^{*} y_{j}}-\frac{1}{\left(1+\lambda_{2}^{*} y_{j}\right)^{2}}\right) e^{-\left(1+\lambda_{2}^{*} y_{j}\right) g_{0 j}}\right]_{0}^{\infty} .
\end{aligned}
$$

\section{REFERENCES}

[1] M. G. Khoshkholgh, K. Navaie, and H. Yanikomeroglu, "Impact of the primary network activity on the maximum achievable capacity of DSCDMA/OFDM spectrum sharing," in Proc. IEEE 68th Veh. Technol. Conf. VTC-2008 Fall, Calgary, Canada, Sept. 2008.

[2] "Spectrum policy task force report," Federal Communications Commission, Tech. Rep., Nov. 2002 [Online]. Available: http://hraunfoss.fcc.gov/edocs_public/attachmatch/DOC-228542A1.pdf.

[3] J. M. Peha, "Approaches to spectrum sharing," IEEE Commun. Mag., pp. 10-12, Feb. 2005.

[4] Q. Zhao and B. Sadler, "A survey of dynamic spectrum access: signal processing, networking, and regulatory policy," IEEE Signal Processing Mag., vol. 24, no. 3, pp. 79-89, May 2007.

[5] X. Liu and S. N. Shankar, "Sensing-based opportunistic channel access," ACM J. Mobile Networks Applications (MONET), vol. 11, no. 4, pp. 577-591, Aug. 2006

[6] M. Gastpar, "On capacity under receive and spatial spectrum-sharing constraints," IEEE Trans. Inform. Theory, vol. 53, no. 2, pp. 471-487, Feb. 2007.

[7] S. A. Jafar and S. Srinivasa, "Capacity limits of cognitive radio with distributed and dynamic spectral activity," IEEE J. Select. Areas Commun., vol. 25 , no. 3, pp. 529-537, Apr. 2007.

[8] A. Ghasemi and E. S. Sousa, "Fundamental limits of spectrum-sharing in fading environments," IEEE Trans. Wireless Commun., vol. 6, no. 2, pp. 649-658, Feb. 2007.

[9] Y. Chen, Q. Zhao, and A. Swami, "Joint design and separation principle for opportunistic spectrum access in the presence of sensing errors," submitted to IEEE Trans. Inform. Theory, Feb. 2007 [Online]. Available: http://www.ece.ucdavis.edu/ qzhao/Journal.html.

[10] Q. Zhao, L. Tong, A. Swami, and Y. Chen, "Decentralized cognitive MAC for opportunistic spectrum access in ad hoc networks: a POMDP framework," IEEE J. Select. Areas Commun., vol. 25, no. 3, pp. 589-600, Apr. 2007.

[11] H. Su and X. Zhang, "Cross-layer based opportunistic MAC protocols for QoS provisionings over cognitive radio wireless networks," IEEE J. Select. Areas Commun., vol. 26, no. 1, pp. 118-129, Jan. 2008.

[12] S. Haykin, "Cognitive radio: brain-empowered wireless communications," IEEE J. Select. Areas Commun., vol. 23, no. 2, pp. 201-220, Feb. 2005.

[13] Y. Xing and R. Chandramouli, "Distributed discrete power control in wireless data networks using stochastic learning," in Proc. IEEE 38th Annual Conf. Inform. Sciences Systems (CISS'04), Princeton, NJ, Mar. 2004.

[14] "Establishment of an interference temperature metric to quantify and manage interference and to expand available unlicensed operation in certain fixed, mobile and satellite frequency bands (ET Docket no. 03237)." FCC Tech. Rep., May 2007.

[15] D. Tse and P. Viswanath, Fundamentals of Wireless Communication. Cambridge University Press, 2004.

[16] A. J. Viterbi, CDMA: Principles of Spread Spectrum Communication. Addison-Wesley, 1995.

[17] K. Navaie and A. R. Sharafat, "A framework for UMTS air interface analysis," Canadian J. Electrical Computer Engineering, vol. 28, no. 2, pp. 113-129, July/Oct. 2003.

[18] A. J. Goldsmith, Wireless Communications. Cambridge University Press, 2005.

[19] Y. C. Liang, Y. Zeng, E. C. Y. Peh, and A. T. Hoang, "Sensingthroughput tradeoff for cognitive radio networks," IEEE Trans. Wireless Commun., vol. 7, no. 4, pp. 1326-1337, Apr. 2008.



Mohammad G. Khoshkholgh received his B.Sc. degree in Electrical Engineering from Isfahan University, Isfahan, Iran, in 2006, his M.Sc. degree in Electrical Engineering from the Tarbiat Modares University, Tehran, Iran, in 2008. He is currently working towards the Ph.D. degree in Tarbiat Modares University, Tehran, Iran. His research focus is on radio resource allocations, and spectrum sharing.

Thus (24) is obtained. 


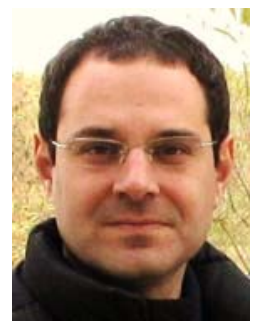

Keivan Navaie (S'02-M'05) received his B.Sc. degree from Sharif University of Technology, Tehran, Iran, his M.Sc. degree from the University of Tehran, Tehran, Iran, and his Ph.D degree from Tarbiat Modares University, Tehran, Iran, all in Electrical Engineering in 1995, 1997 and 2004 respectively.

From March to November 2004, he was with the School of Mathematics and Statistics, Carleton University, Ottawa, Canada, as a Postdoctoral Research Fellow. From December 2004 to September 2006, he was with the Broadband Communication and Wireless System (BCWS) Centre, Carleton University, Ottawa, Canada where he was the project manager of BCWS participation in European Union 6th Framework integrated project, the Wireless World Initiative New Radio (WINNER) on beyond 3G wireless systems. Since September 2006 he has been an Assistant Professor in the Department of Electrical and Computer Engineering, Tarbiat Modares University, Tehran, Iran.

Dr. Navaie has been a member of Technical Committee of WASA'06, IEEE WCNC'08, 16th Iranian Conference on Electrical Engineering (ICEE'07), IEEE GlobeCom'08, IEEE WiMob'08, IEEE WCNC'09, and CNSR'09. His research interests are mainly in the resource management issues of cellular and multi-hop wireless networks, cooperative communications, spectrum sharing and traffic modelling. He is a member of the IEEE.

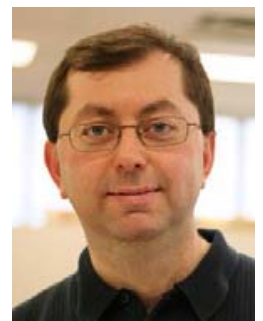

Halim Yanikomeroglu received a B.Sc. degree in Electrical and Electronics Engineering from the Middle East Technical University, Ankara, Turkey, in 1990, and a M.A.Sc. degree in Electrical Engineering (now ECE) and a Ph.D. degree in Electrical and Computer Engineering from the University of Toronto, Canada, in 1992 and 1998, respectively. He was with the R\&D Group of Marconi Kominikasyon A.S., Ankara, Turkey, from 1993 to 1994.

Since 1998 Dr. Yanikomeroglu has been with the Department of Systems and Computer Engineering at Carleton University, Ottawa, where he is now an Associate Professor with tenure. Dr. Yanikomeroglu's research interests cover many aspects of the physical, medium access, and networking layers of wireless communications with a special emphasis on multihop/relay/mesh networks and cooperative communications. Dr. Yanikomeroglu's research is currently funded by Samsung (SAIT, Korea), Huawei (China), Communications Research Centre of Canada (CRC), Nortel, and NSERC.

Dr. Yanikomeroglu has been involved in the steering committees and technical program committees of numerous international conferences; he has also given 16 tutorials in such conferences. Dr. Yanikomeroglu is a member of the Steering Committee of the IEEE Wireless Communications and Networking Conference (WCNC), and has been involved in the organization of this conference over the years, including serving as the Technical Program Co-Chair of WCNC 2004 and the Technical Program Chair of WCNC 2008.

Dr. Yanikomeroglu was an editor for IEEE TRANS ACTIONS ON WIRELESS COMMUNiCATIONS [2002-2005] and IEEE COMMUNiCATIONS SuRveYs \& TUTORIALS [2002-2003], and a guest editor for WILEY JOURNAL ON Wireless CoMmunications \& MobiLe Computing. He was an Officer of IEEE's Technical Committee on Personal Communications (Chair: 200506, Vice-Chair: 2003-04, Secretary: 2001-02), and he was also a member of the IEEE Communications Society's Technical Activities Council (2005-06). Dr. Yanikomeroglu is an adjunct professor at King Saud University, Riyadh, Saudi Arabia; he is also a registered Professional Engineer in the province of Ontario, Canada. 